megadalton transmissible plasmid, ' was isolated in the United States of America. $^{2}$ These strains of TRNG were found in England and the Netherlands in $1988 .^{34}$

We now present the first TRNG isolate in Spain, from a prostitute woman from Madrid; she was treated with spectinomycin. The isolate of $N$. gonorrhoeae was resistant to tetracycline (Minimal Inhibitory Concentration $16 \mathrm{mg} / \mathrm{l}$ ), sensitive to penicillin (MIC $0.06 \mathrm{mg} / \mathrm{l}$ ), spectinomycin (MIC $16 \mathrm{mg} / \mathrm{l}$ ) and ceftriaxone (MIC $0.0015 \mathrm{mg} / \mathrm{l}$ ), and moderately sensitive to cefoxitin (MIC 0.5 mg/1).

The plasmid analysis ${ }^{5}$ shows two plasmids, of 25.2 and 2.6 megadaltons; digestion of plasmid deoxyribonucleic acid with Hinc II and Sma I shows a band pattern different from the one found when a 24.5 megadalton plasmid (transfer plasmid) was digested.

Auxotype and serotype were determined as has been previously described; ${ }^{6}$ the isolate belonged to class-Pro/Bpyst.

A rapid international spread of TRNG may be occurring as we predicted recently. ${ }^{7}$

JA VAZQUEZ* S BERRÓN* B MENÉNDEZ†

* Servicio de Bacteriologia, Centro Nacional de Microbiologia, Virologia e Inmunologia Sanitarias, Majadahonda (Madrid), Spain †Centro Sanitario Sandoval, Comunidad Autónoma de Madrid, Spain

1 Morse SA, Johnson SR, Biddle JW, Roberts MC. High level tetracycline resistance to Neisseria gonorrhoeae is result of acquisition of streptococcal tet-M determinant. Anti-microb Agents Chemother 1986;30:664-70.

2 Knapp JS, Zenilman JM, Biddle JW, et al. Frequency and distribution in the United States of strains of Neisseria gonorrhoeae with plasmid mediated high level resistance to tetracycline. $J$ Infect Dis 1987;155:819-22.

3 Waugh MA, Lacey CJN, Hawker PM, et al. Spread of Neisseria gonorrhoeae resistant to tetracycline outside the United States of America. Br Med J 1988;296:898.

4 Roberts MC, Van Klingeren B, Wagenvoort JHT, Knapp JS. Tet$M, \beta$-lactamase containing Neisseria gonorrhoeae (TRNG/PPNG) in the Netherlands. Antimicrob Agents Chemother 1988;32:158.

5 Birboim HC, Doly A. A rapid alkaline extraction procedure for screening recombinant plasmid DNA. Nucleic Acids Res 1979;7:1513-23.
6 Fenoll A, Berrón S, Vázquez JA. Analysis of penicillinase producing Neisseria gonorrhoeae isolates in Madrid (Spain) from 1983-85. Epidemiol Infect 1987;99:455-62.

7 Vázquez JA. Infección gonocócica. Ministerio de Sanidad, Boletin Microbiológico Semanal 1988;12/88: $1-4$.

\section{Workload in genitourinary medicine clinics in England}

One of the criteria used to assess workloads in genitourinary clinics is based on the reports of clinic attendances, that is, new cases, return visits and diagnoses. Yet there does not appear to be an agreed uniform approach to calculating these figures between different clinics, making comparisons of workloads difficult to interpret.

When to reregister patients (and thus create a new visit and a new diagnosis) is an area open to individual interpretation. A dilemma often arises in cases of chronic or recurrent conditions like candidiasis, recurrent nonspecific urethritis, genital warts, etc.

Different clinic practices may also contribute to false impressions of workload. For example, considering the fact that many of our patients are employed and/or have young children, we operate a fairly liberal policy of allowing patients to phone for results where the doctor considers this appropriate. This obviously creates a lot of work for reception staff, who still have to retrieve case notes from filing and locate results, and often have to ask a doctor either for a comment or to talk to the patient. Thus, although we are providing an optimal patient oriented facility much of this work is not included in assessment of workload for the clinic. Conversely, a clinic that insists on a visit for results will show higher clinic attendance figures.

A clinic, like ours, that provides a full colposcopy service through to cold coagulation, loop excision or laser therapy, hardly has its workload adequately represented by a single diagnosis of $\mathrm{Cl1}$ when the patient attends with genital warts and is subsequently managed by us for an abnormal smear. A clinic that does not even do cytology would apparently have a similar workload to us - based on diagnoses alone.

With the imminent National Health Service review and an apparent decrease in clinic attendance numbers, despite increased workload due to the complexity of the current conditions,' there is pressure on clinics to institute measures to get numbers to reflect the work being done. In future it is likely that greater credit and resources will be given to clinics that show high new patient attendance numbers and a high new patient to follow up attendance ratio. To our knowledge no consensus on performance indicators in genitourinary medicine has yet been reached but attendance and ratios are likely to be major considerations. It is essential that our practice is represented in a comprehensive, accurate and work sensitive way to reflect true workload and changing trends. Clinic variance in registration and reregistration policies will make comparisons inaccurate and regional planning difficult. COLM O'MAHONY DEREK J TIMMINS University Department of Genitourinary Medicine, Royal Liverpool Hospital, Prescot Street,

Liverpool L7 8XP, UK

1 Thin RN. Workloads in genitourinary medicine clinics in England. Genitourin Med 1989;65:376-81.

\section{Syphilis among heterosexuals}

We read with interest the paper by Dr van den Hoek and colleagues ${ }^{i}$ and have therefore performed a survey to determine whether acquisition of syphilis at our unit is associated with prostitution or drug abuse.

The case notes of all patients attending our clinic between 1985 and 1989 with primary, secondary or early latent syphilis (less than one year's duration) were reviewed retrospectively. The following clinical details were recorded: sex; sexual orientation; history of prostitution; history of drug abuse; whether infection contracted in UK or abroad. These details are summarised in the table.

No patients gave a history of prostitution or drug abuse. As has been noted in other studies the number of homo/bisexual men with infectious syphilis has greatly decreased and the proportion of heterosexuals with infectious syphilis is increasing. But there has been no increase in the number of heterosexuals with syphilis and an increasing proportion of 
Table Infectious syphilis at St. Mary's hospital department of genito-urinary medicine 1985-1989

\begin{tabular}{lccccc}
\hline & 1985 & 1986 & 1987 & 1988 & 1989 \\
\hline Heterosexual men & 14 & 2 & 10 & 12 & 6 \\
Homo/bisexual men & 65 & 25 & 23 & 11 & 10 \\
Women & 6 & 2 & 9 & 9 & 5 \\
Total & 85 & 29 & 42 & 32 & 21 \\
$\begin{array}{l}\text { Percentage infected abroad } \\
\text { (where geographical location of }\end{array}$ & $22 \%$ & $26 \%$ & $38 \%$ & $57 \%$ & $71 \%$ \\
infection recorded) & & & & & \\
\hline
\end{tabular}

patients give a history suggesting infection abroad. Some units in London, however, have reported a recent resurgence of infectious syphilis among heterosexuals. ${ }^{2}$

Although the results of this retrospective survey must be treated with caution it seems there is no link between infectious syphilis and prostitution in patients attending this unit.

Drug abuse and prostitution are strongly associated with the current heterosexual syphilis epidemic in the USA and this carries with it the risk of increasing HIV transmission within this group. ${ }^{3}$ Prospective studies are needed to assess the significance of this phenomenon in the UK.
PD FRENCH

J DAVIS

D GOLDMEIER

Department of Genito-urinary Medicine, Jefferiss Wing, St Mary's Hospital, Praed St, London W2 INY, UK

1 van den Hoek JAR, van der Linten MMD, Coutinho RA. Increase of infectious syphilis among heterosexuals in Amsterdam: its relationship to drugs and prostitution. Genitourin Med 1990;66:31-32.

2 Tang A, Barlow D. Resurgence of heterosexually acquired early syphilis in London. Lancet 1989;ii: 166.

3 Anonymous. Relationship of syphilis to drug use and prostitution-Connecticut and Philadelphia, Pennsylvania. MMWR 1988;49:755-64.
MATTERS ARISING

Treatment of external genital warts

Stone and colleagues state that the patient should be carefully positioned on the ground pad for electrodesiccation. Using the monoterminal technique (as when using the hyfrecator) it is not necessary to use the ground pad. In fact electrodesiccation is usually described as a monoterminal technique.

K SANKAR K SANKAR
Newcastle General Hospital,
Westgate Road, Newcastle upon Tyne $N E 46 B E$, UK

1 Stone KM, Becker TM, Hadgu A, Kraus SJ. Treatment of external genital warts: a randomised clinical trial comparing podophyllin, cryotherapy, and electrodesiccation. Genitourin Med 1990;66:16-9.
Genital warts and the need for screening

An interesting controversy has arisen concerning the benefit of screening for concomitant sexually transmitted diseases (STD) in patients who present to genitourinary medicine departments with genital warts. Malcolm Griffiths ${ }^{1}$ argues that such screening of women is of limited value on the evidence of a relatively low prevalence of genital infection with Chlamydia trachomatis and other STDs in his patient population. More recently, Carne and Dockerty $^{2}$ have confirmed the traditional view that screening is mandatory. Neither paper, however, distinguishes between patients whose sole symptom was genital warts and patients with additional genital symptoms which warrant screening for genital infection on their own account. This latter group might be anticipated to have a high rate of coinfection.

During the period April to September 1989, first presentations of genital warts were seen in 297 women and 249 men attending this genitourinary medicine department. All were screened for Neisseria gonorrhoeae, Chlamydia trachomatis (by culture in men, ELISA in women), urethritis, Candida albicans, Trichomonas vaginalis and bacterial vaginosis using standard techniques. Of these patients, $138(46 \%)$ women and 52 $(21 \%)$ men had coincidental genital symptoms necessitating full genital screening. Of the 356 patients whose sole symptom was genital warts, 69 $(33 / 159,21 \%$ women $(F)$ and $36 / 197$, $18 \%$ men $(M))$ were found to have asymptomatic coinfection with a sexually transmitted disease, comprising gonorrhoea (1M, 5F), chlamydia (10M, 19F), non-gonococcal urethritis (22M), trichomoniasis (9F) and genital herpes (3M). Our finding of asymptomatic coinfection with a STD in one fifth of patients whose sole symptom was genital warts confirms the importance of screening for coinfection.

Genital infection with Chlamydia trachomatis or Neisseria gonorrhoeae are infections of significant morbidity, particularly in women and every opportunity for screening the multipartner sexually active population should be encouraged. Rather than reduce screening of patients with genital warts as advocated by Griffiths we would welcome an expansion of screening for asymptomatic genital infection in gynaecology, general practice and family planning settings, a secondary preventative measure constrained by resources and professional resistance.

$$
\begin{array}{r}
\text { D T JAYAWEERA } \\
\text { I H AHMED } \\
\text { C J BIGNELL } \\
\text { K E ROGSTAD } \\
\text { Perth and Amberley House Clinics, } \\
\text { Postern Street, } \\
\text { General Hospital, } \\
\text { Nottingham NG1 6HA, UK }
\end{array}
$$

1 Griffiths M. Genital warts and the need for screening. Genitourin Med 1989:65:399.

2 Carne CA, Dockerty G. Genital warts: need to screen for coinfection. Br Med J 1990;300:459.

\section{Genital infections due to Neis- seria meningitidis}

SIR,-In a recent report Wilson et al ${ }^{\prime}$ drew attention to a case of urethritis in a heterosexual male caused by Neisseria meningitidis probably acquired by 